\title{
Non-Thermal Atmospheric Dielectric Bamier Discharge Plasma, Medical Application Studies in Thailand
}

\author{
Chanchai Chutsirimongkol,,$^{\mathrm{a},{ }^{*}}$ Dheerawan Boonyawan, ${ }^{\mathrm{b}}$ Niwat Polnikorn, ${ }^{\mathrm{c}}$ \\ Wasini Techawatthanawisan, ${ }^{d}$ Treenuch Kundilokchai, ${ }^{d}$ Chayanid Bunsaisup, ${ }^{d}$ \\ Paisal Rummaneethorn, ${ }^{d}$ Wicharn Kirdwichai, ${ }^{e}$ Apirag Chuangsuwanich, ${ }^{\dagger}$ \\ \& Pannapa Powthongg \\ aThailand Center of Excellence for Life Sciences, Bangkok, 10400, Thailand/PhotoBioCare \\ Co., Ltd., Nonthaburi 11120, Thailand; ' $C$ iang Mai University, Chiang Mai 50200, Thailand; \\ 'Kasemrad Hospital, Bangkok 10240, Thailand; 'Mae Fah Luang University, Bangkok 10110, \\ Thailand; eBurapha University, Chonburi 20130, Thailand; 'Surgery Department, Siriraj \\ Hospital, Mahidol University, Bangkok 10700, Thailand; '9Rangsit University, Pathum Thani \\ 12000, Thailand

\footnotetext{
*Address all correspondence to: Chanchai Chutsirimongkol, PhotoBioCare Co., Ltd., 224/1, Victoria Building M9,
} \\ Pakkred District, Nonthaburi 11120, Thailand, E-mail: chanchai@pbc.co.th, info@pbc.co.th
}

\begin{abstract}
Atmospheric Dielectric Barrier Discharge (DBD) plasma development is one of the most interesting subjects to our group. Fridman et al. ${ }^{*}$ has concluded that Floating DBD Direct Plasma are more effective in bactericidal property, it contains higher free charged particles than indirect type. DBD plasma sources can provide low temperature or non-thermal (cold) plasmas, and these plasmas can be created with ambient air or with a noble gas, such as helium or argon, as the carrier medium. It can be used with an electrode as a contact or noncontact method to target a tissue. However, the drawback of direct DBD plasma is that it will work only in short gap distances, on the order of a few millimeters. Our group has investigated the clinical safety and efficacy of a novel DBD non-thermal atmospheric plasma source (BIOPLASMA Cell Modulation: PhotoBioCare, Thailand). The major area has been focused on its bactericidal disinfection property, application in common dermatologic problems, in skin aesthetic facial rejuvenation and chronic wound management, from 2012 to 2016.
\end{abstract}

KEY WORDS: non-thermal atmospheric plasma, DBD, dielectric barrier discharge plasma source, medical application outcome

\section{INTRODUCTION}

Many potential benefits of atmospheric non-thermal plasma applications are widely recognized as an interesting emerging technology in medicine. Technically, they allow us to modulate chemical changes in biomolecules at atomic and molecular levels and control thermal injury by matching the appropriate level to the tissue target. This leads to consequence of plasma tissue interaction inducing changes at cellular and subcellular level.

*Fridman G, Brooks AD, Balasubramanian M, Fridman A, Gutsol A, Vasilets VN, Ayan H, Friedman G. Comparison-pressure plasma of direct and indirect effects of non-thermal atmospheric on bacteria. Plasma Proc Polymer. 2007;4:370-5. 
Many potential clinical applications have been developed during the last few decades and can be grouped in three major areas:

1. Broad-spectrum microbial and biological decontamination

2. New surface property modification of living and nonliving objects

3. Direct therapeutic application

Among the different types of atmospheric plasma generator sources, our group in Thailand has primarily focused on the dielectric barrier discharge (DBD) method to produce low temperature or tissue tolerable thermal plasma. Our team began our basic studies of the system (BIOPLASMA Cell Modulation: PhotoBioCare Thailand) on its safety and efficacy and evaluated its potential clinical applications during the last 5 years (Fig. 1). The outcome of the studies prove its potential in bactericidal disinfection, dermatology, skin aesthetics, and chronic wound healing management application.

\section{MATERIALS AND METHODS}

A novel atmospheric plasma generator source (BIOPLASMA Cell Modulation system) has an adjustable power level on a 1-10 scale and adjustable repetitive rate from 10 $110 \mathrm{~Hz}$. It delivers pulses of high voltage (4-7 kV) with low milliamp current in high frequency alternating current $(16-27 \mathrm{kHz})$. Special designs of DBD electrodes allow power output in the range of a few hundred milliwatts to a few watts. The system can be used as contact or noncontact to skin or other target tissues. The DBD microfilament plasma beam contains various active elements that directly interact with the target. Different types of gas, either room air or noble gas such as helium and argon, can be used as the carrier gas. Different types of beam characteristics can be designed and created to match various potential indications. Application can be static or dynamic movement in various scan motion techniques adjusted by the operator to obtain the expected outcome according to guideline parameters for each indication. ${ }^{1}$

The system has been evaluated for medical standards device certification approval by Electrical and Electronic Product Testing Center/National Science and Technology Development Agency in Thailand, with Thailand Center of Excellence for Life Science (TCELS) support described elsewhere. Evaluation of maximum skin tolerant dose range to heat and pain of each plasma parameter on volunteer subjects were carried out initially by PhotoBioCare as shown in Fig. 1.

\section{A. Laboratory Analysis}

Electrical analysis and optical spectral analysis of various radicals and plasma parameters was conducted by the Plasma \& Beam Physics Research Facility (PBP), Chiang Mai University (Fig. 2). ${ }^{2,3}$ In vitro plasma interaction with fungus, and bacterial inhibi- 


\section{Clinical safety evaluation of plasma skin tolerant dose}
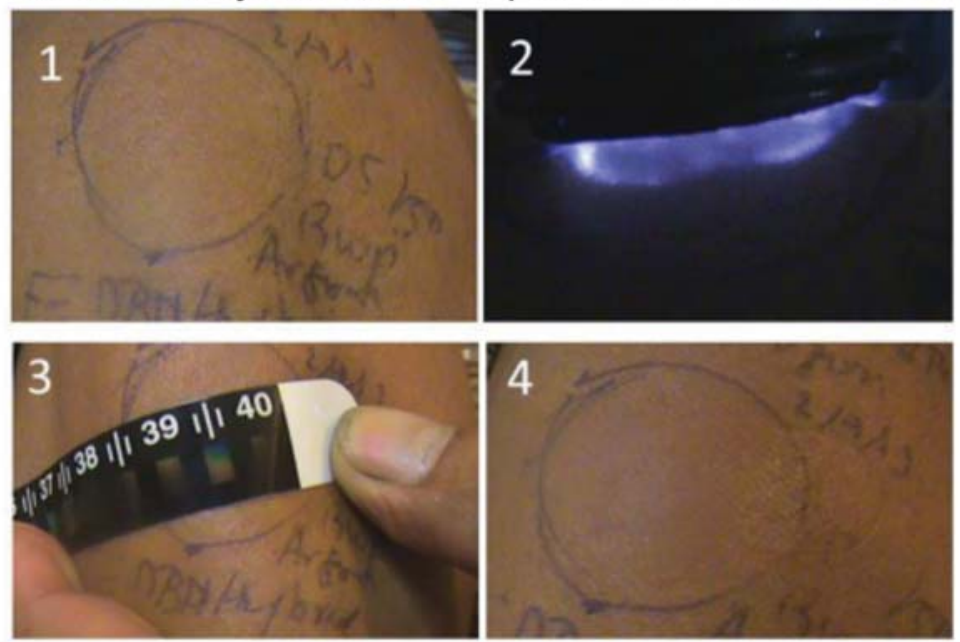

Hybrid DBD FE Bioplasma Argon jet setting $05 / 50 \mathrm{~Hz}$.

(1) Pretreatment; (2) $40 \mathrm{sec}$. maximum tolerant dose, moderate hot and pain;

(3) skin temp $39.5^{\circ} \mathrm{C}$ post-treatment; (4) skin $15 \mathrm{~min}$. later.

\section{Clinical safety evaluation of plasma skin tolerant dose}
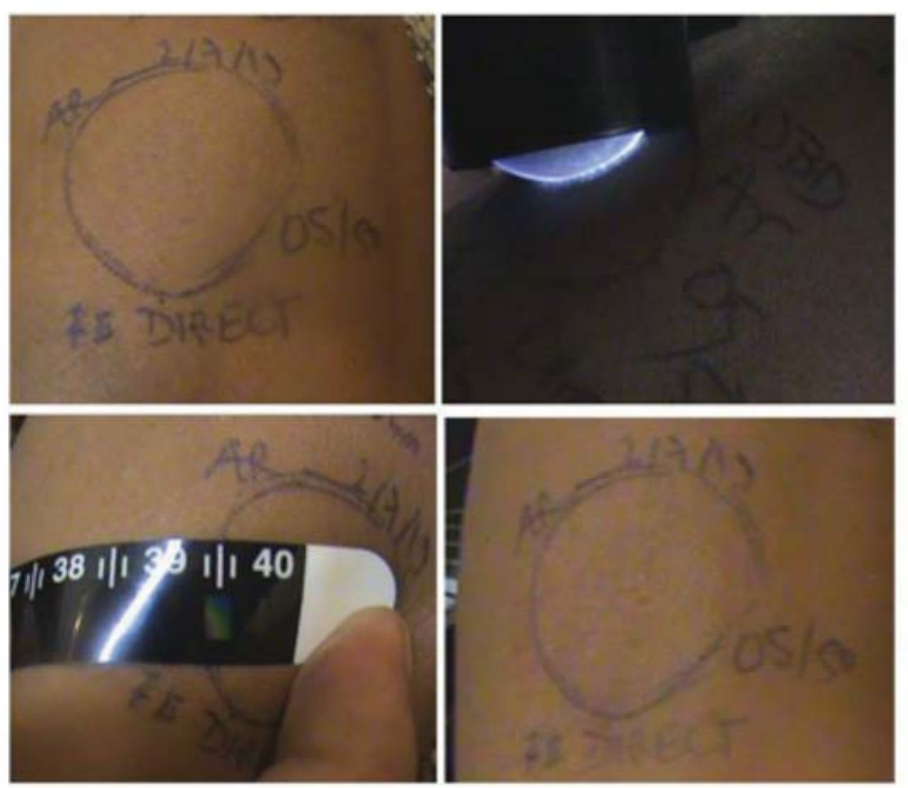

Direct DBD FE Argon 05/50 setting bioplasma $15 \mathrm{sec}$. Rx.

Pre-plasma jet $\mathrm{Rx}$-immediate temperature $30 \mathrm{~min}$. after $\mathrm{Rx}$.

Skin temp $39.5^{\circ} \mathrm{C}$. Moderate hot and pain, mild skin redness.

Warm sensation for few minutes.

FIG. 1: Maximum heat and pain tolerating skin dose test in volunteer subject

Volume 6, Issues 3-4, 2016 


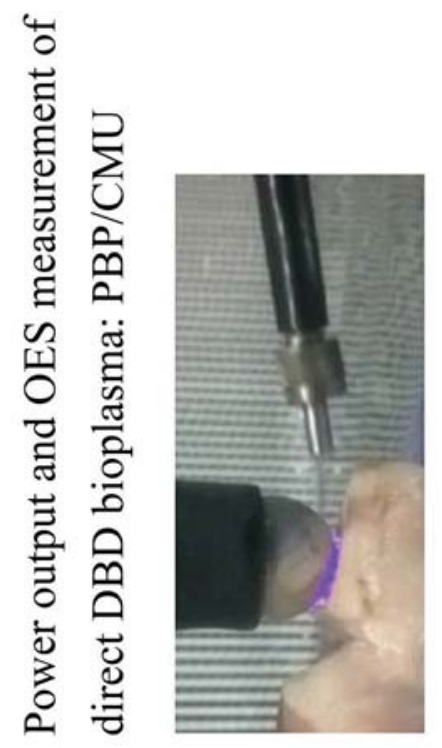

음

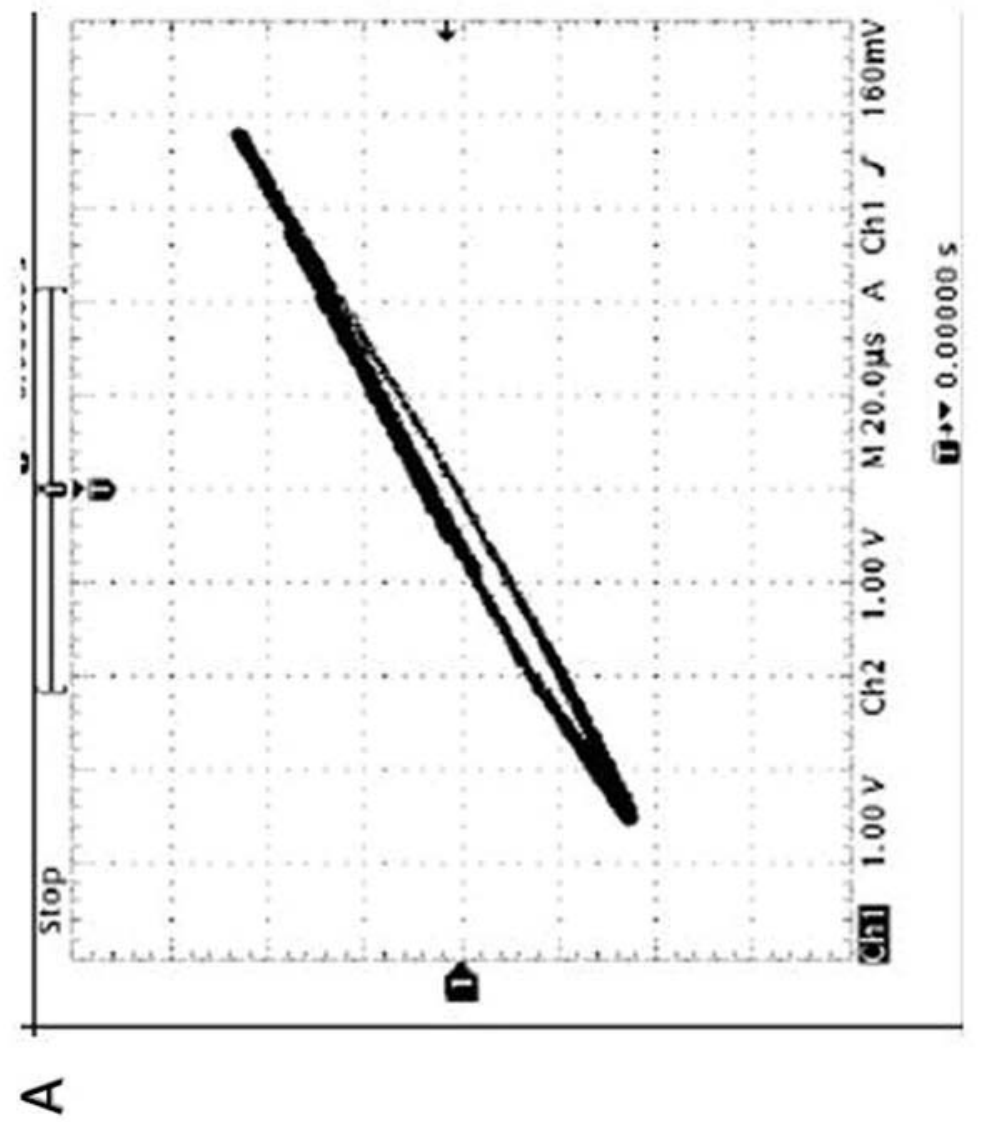

$\underset{0}{\stackrel{0}{0}}$ 


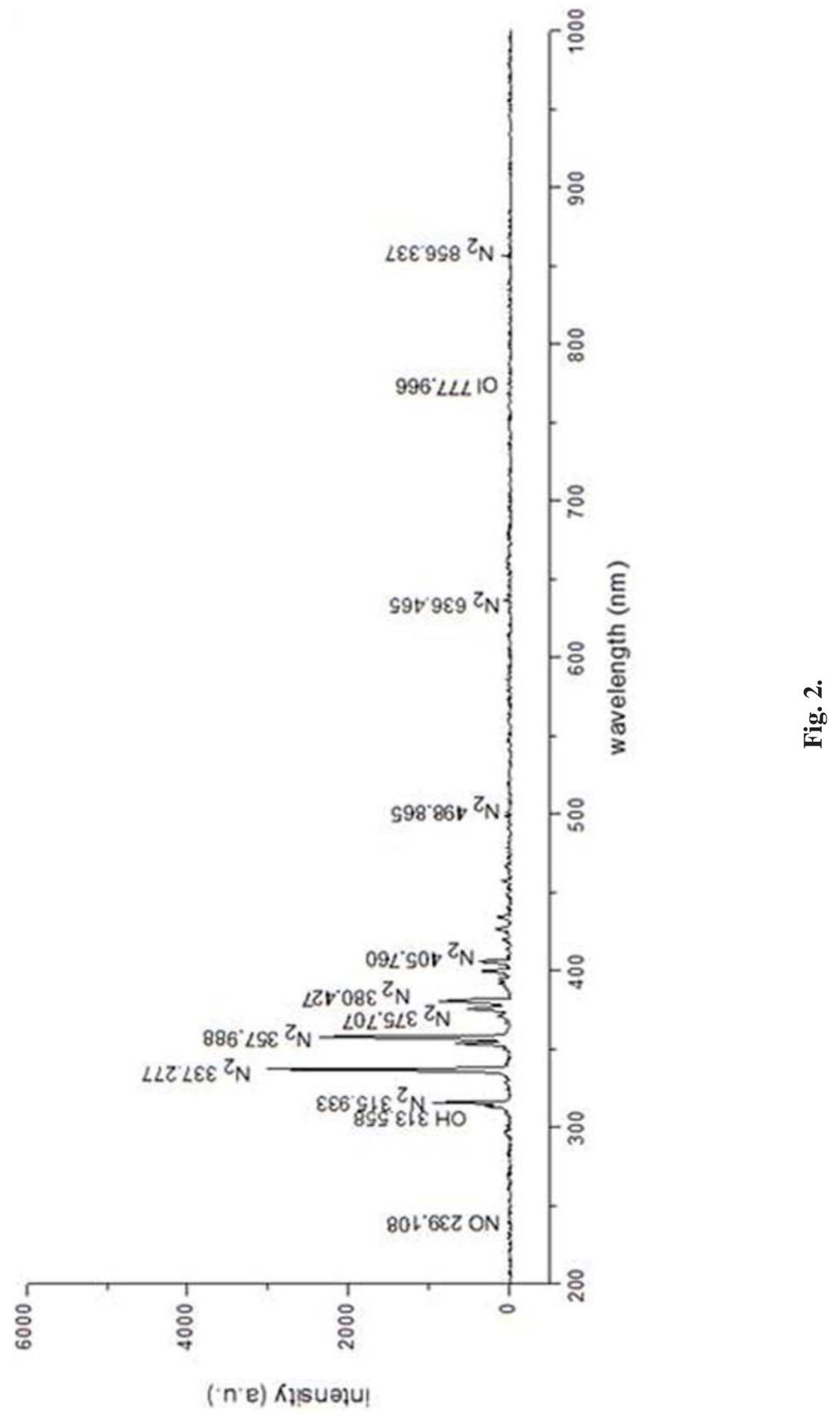

Volume 6, Issues 3-4, 2016 

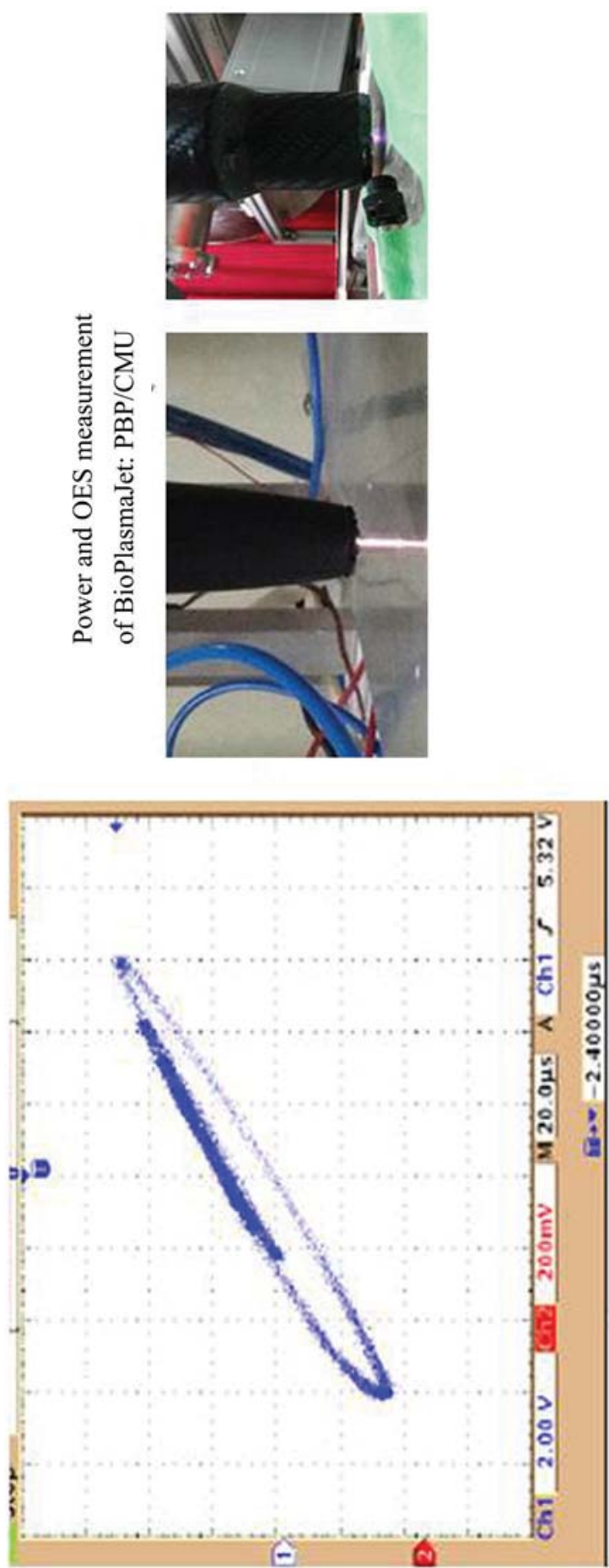

i.

\section{$\infty$}




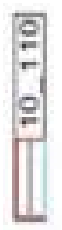

$\stackrel{\ominus}{:}$

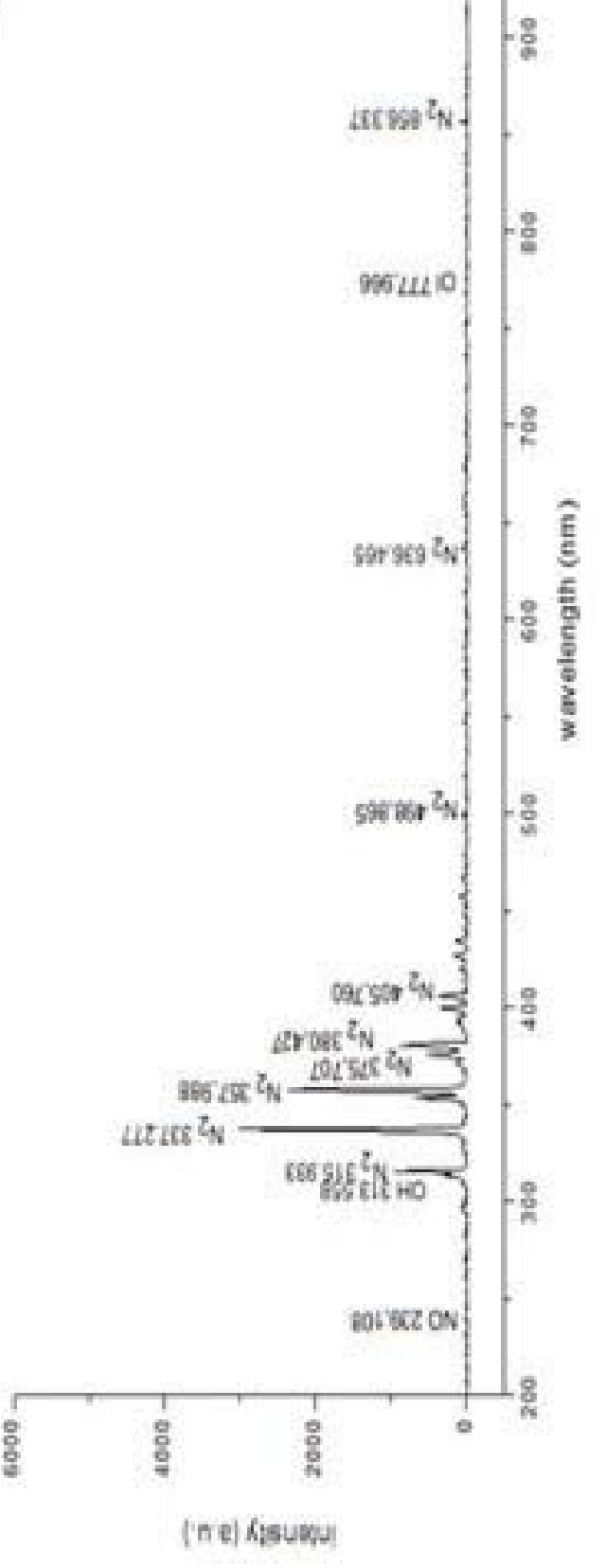


tory and sterilizing dose evaluation were carried out at Chiang Mai University (Fig. 3) and Rangsit University (RSU) (Figs. 4, 5). Maximum doses of skin tolerance to heat and pain of each plasma parameter on volunteer subjects were carried out by PhotoBioCare (PBC), which also evaluated skin etching and plasma skin peeling. Elimination of porphyrin counts, which reflect $P$. acnes (propionibacterium acnes, a Gram-positive anaerobic rod) bacterial skin colonization pre- and post-plasma treatment, was determined with a UV camera by Professor Niwat Polnikorn.

\section{B. Clinical Studies Application of Direct DBD Atmospheric Air Plasma Contact Type}

\section{Acne Vulgaris and Various Postinflammatory Acne Conditions}

The studies carried out by Mae Fah Luang University team and clinical observation by a research team from PhotoBioCare revealed that direct contact DBD Bioplasma creates surface keratin peeling, including removal of dead keratin, keratin plugs in oil gland (comedone), cleaning, and surface decontamination. ${ }^{1}$ In addition, bioplasma is a safe and effective method of reducing $P$. acnes hyper colonization. ${ }^{1}$

Kundilikchai ${ }^{4}$ concluded that it is safe and significantly effective in reducing facial sebum (oil) production, with a sustained effect 4 weeks after discontinuing treatment of 30 cases in a half face control study, once weekly for 4 weeks.

Techawatthanawisan ${ }^{5}$ concluded that it is quite safe and significantly effective for mild and moderate acne, especially inflammatory acne on 31 cases in a study where participants were treated once weekly for 6 weeks.

Bunsaisup and Rummaneethorn ${ }^{6}$ concluded that Bioplasma treatment combined with topical treatment (retinoic acid cream) could enhance the therapeutic efficacy in treatment of moderate facial acne vulgaris, with duration of improvement and satisfaction score comparable to topical treatment alone. This study included half face Bioplasma treatment of 30 Thai volunteers evaluated by Visia Complexion Analysis during once weekly treatment for 4 weeks with a 3 -month follow-up. ${ }^{6}$

The clinical evaluation in both short and long term revealed safe and effective outcome on treatment of acne and acne complication (Fig. 6).

\section{Skin Mycosis Infection}

See Fig. 7.

\section{Aesthetic Skin Appearance Improvement, Plasma Skin Rejuvenation}

Plasma peeling helps clean the skin surface, reduces oily skin, and removes unhealthy skin and skin contamination. This skin exfoliation helps stimulate new skin replacement, and it has a rejuvenating effect. Direct microplasma beams help improve skin 


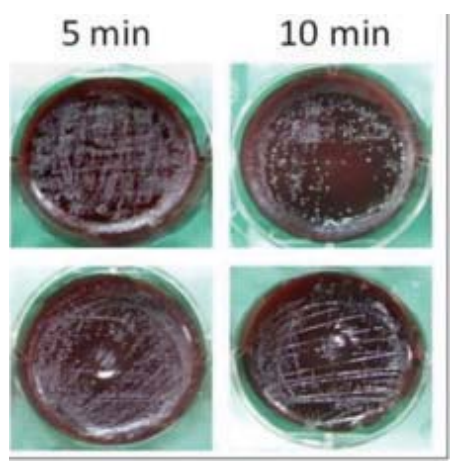

CAP He Jet Needle: E.Faecalis Scan mode (upper) and static mode (lower)

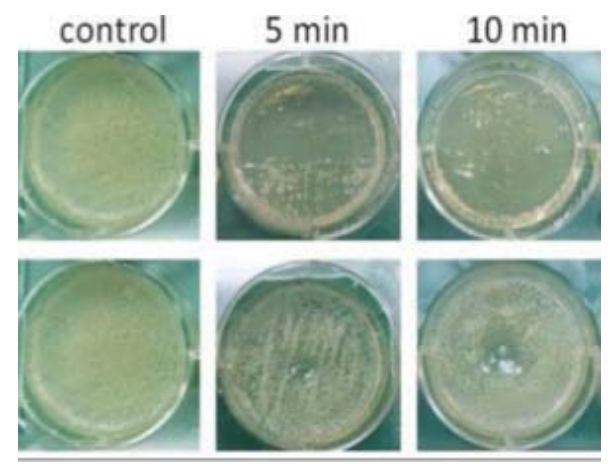

CAP He Jet Needle: C.albicans. Scan mode (upper) and static mode (lower)

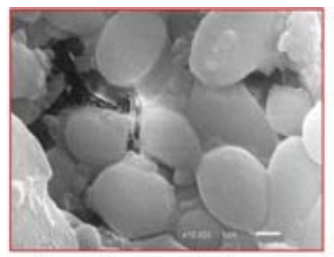

- Negative control

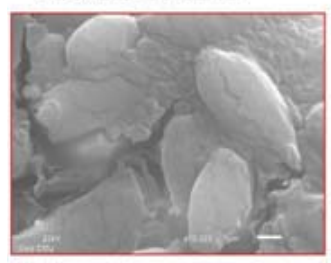

-10-min plasma

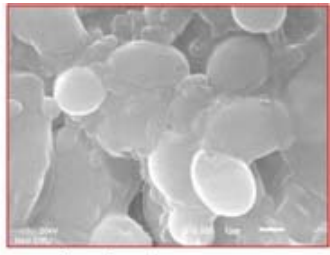

-5-min plasma

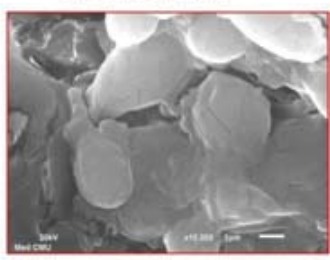

-10-min plasma

Electron microscope evaluation of C.albicans after plasma jet exposure.

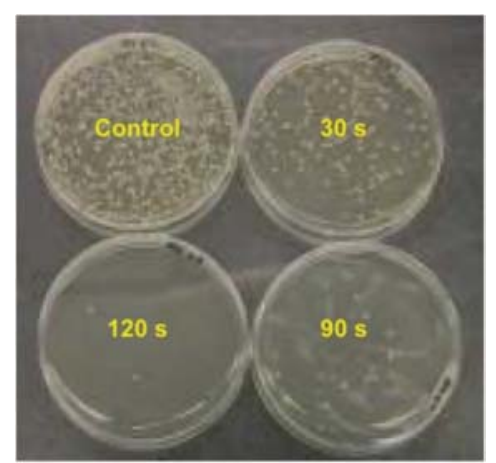

FIG. 3: Study of microbiology exposure to bioplasma jet (PBP CMU). Picture show MRSA respond to various dose of FE DBD Argon Bioplasma Jet. Scan motion over the entire wide area produces a larger microbial eradication zone than fixed spot exposure.

Volume 6, Issues 3-4, 2016 


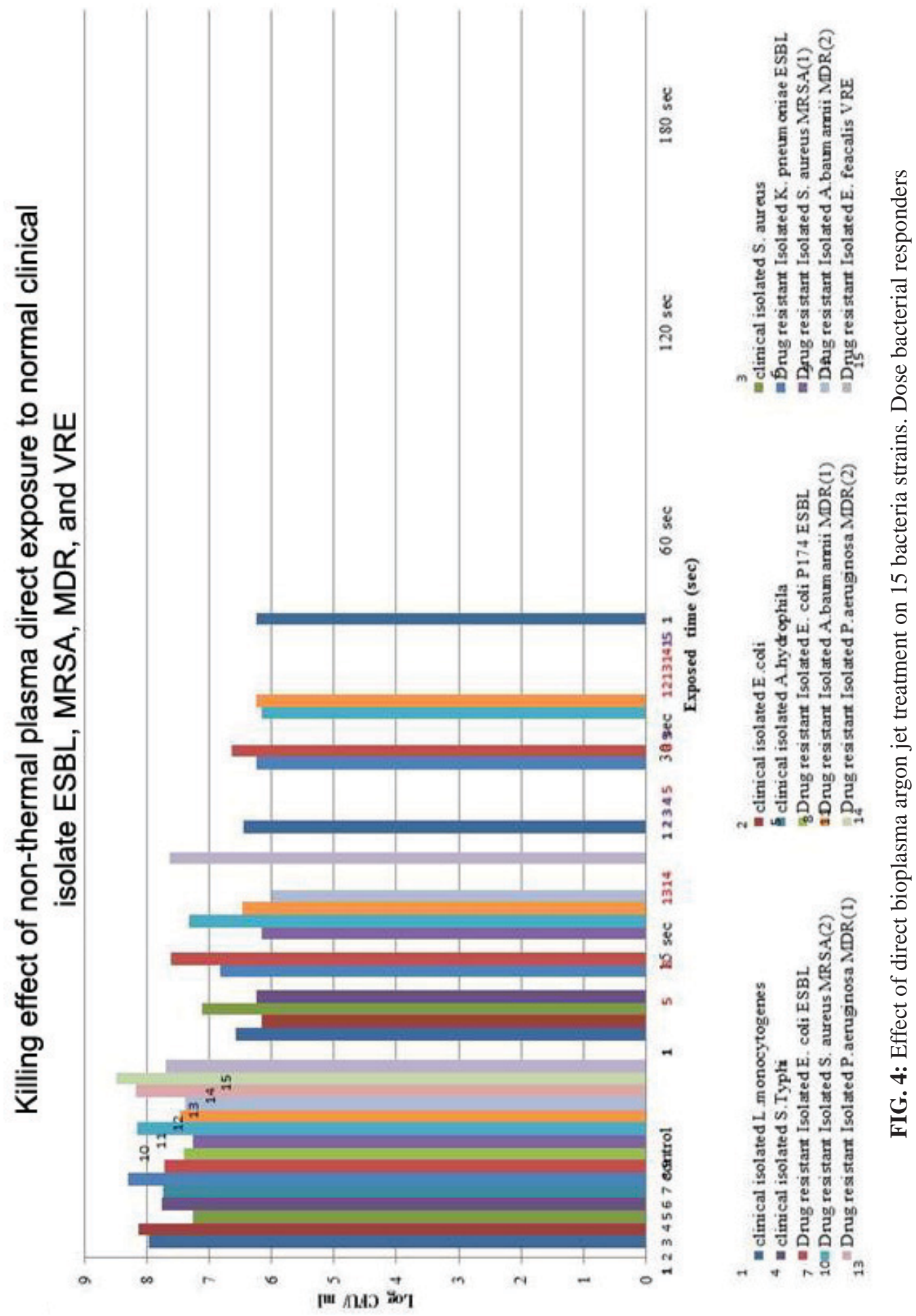




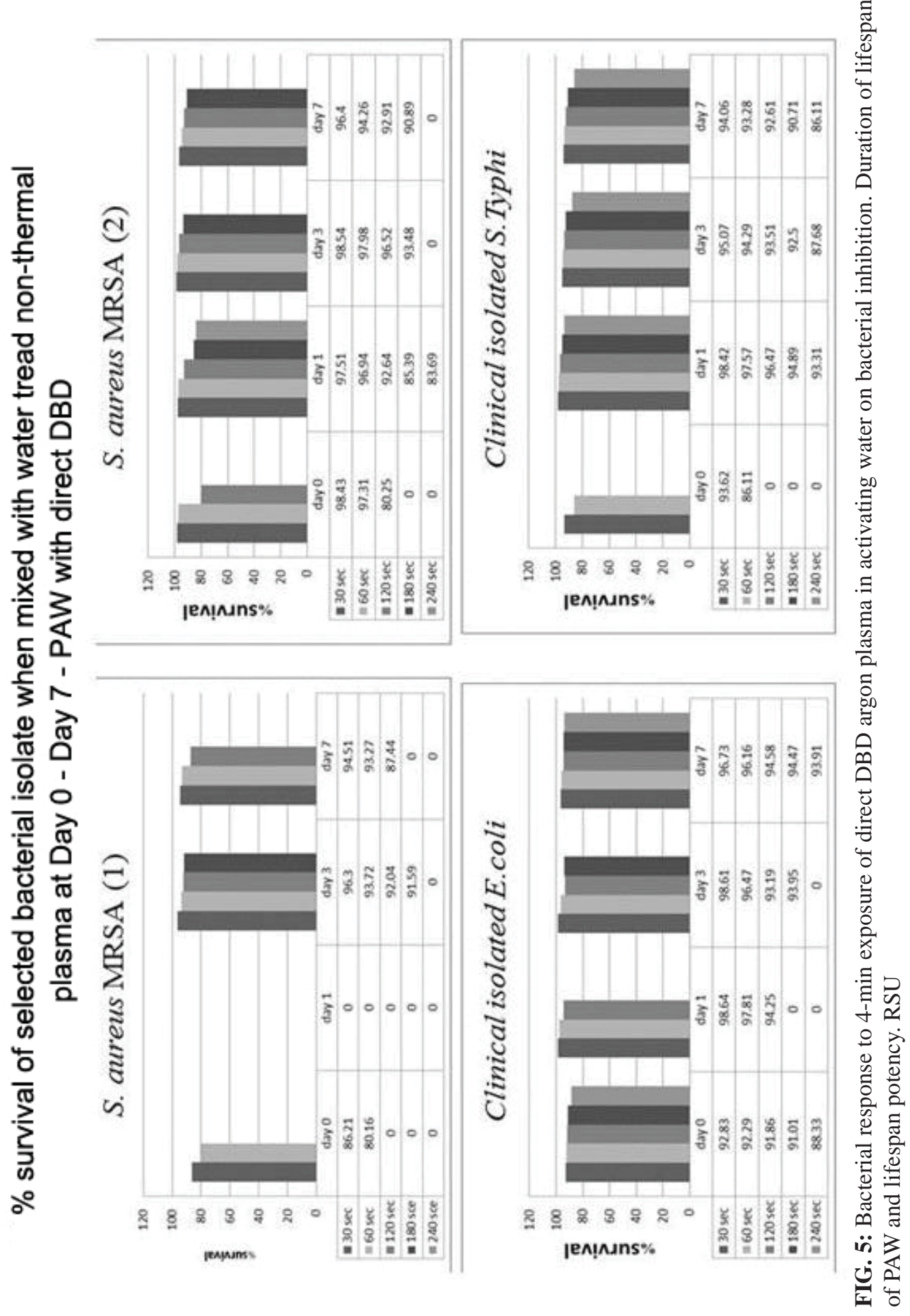

Volume 6, Issues 3-4, 2016 

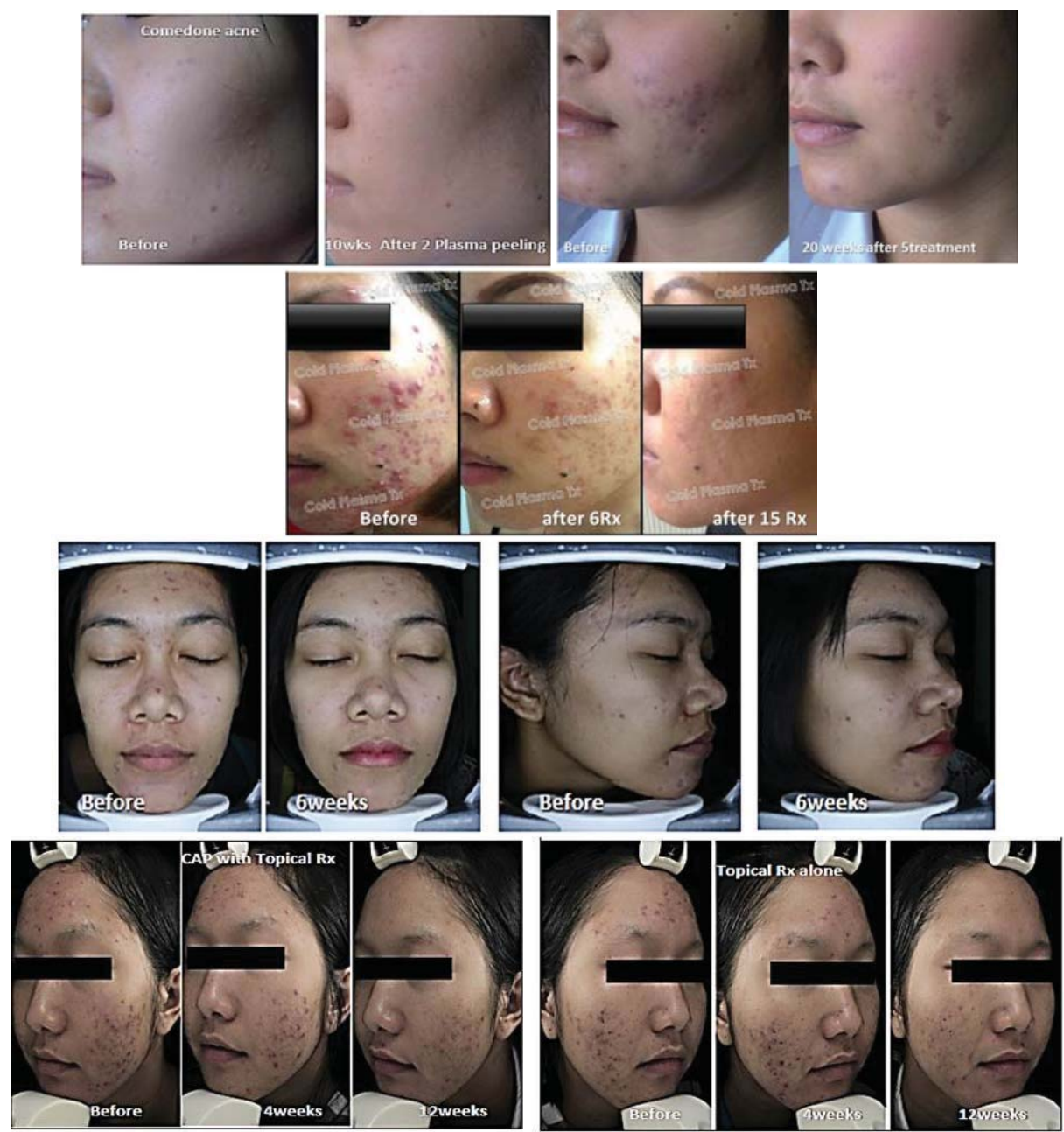

Clinical studies on non-thermal direct BDB plasma for acne

1. The effectiveness of a non-thermal plasma device for reducing sebum production on oily face: half face study of 30 cases once weekly Rx for 4 weeks. ${ }^{4}$

Conclusion: Plasma device is effective in reducing sebum production on oily face with sebumeter measurements and lasts for at least 1 month after in 30 cases half face study.

2. A comparative study on the efficacy of non-thermal plasma device combined with topical treatment versus topical treatment alone for moderate facial acne vulgaris (bottom picture). ${ }^{6}$ Conclusion: Non-thermal plasma combined with otpical treatment could enhance the therapeutic efficiency in treatment of moderate facial acne vulgaris in term of duration of improvement and satisfactory score compared to topical treatment alone.

3. An efficacy assessment of a dielectric barrier discharge plasma device for adjunctive therapy of acne vulgaris: a pilot study of 31 cases, 20 min. once weekly for 6 weeks (middle picture). ${ }^{5}$ Conclusion: Plasma device does appear to have some role in the management of acne; may be beneficial for the treatment of selected group of mild-to-moderate acne patients.

FIG. 6: Outcome of acne treatment and clinical studies 


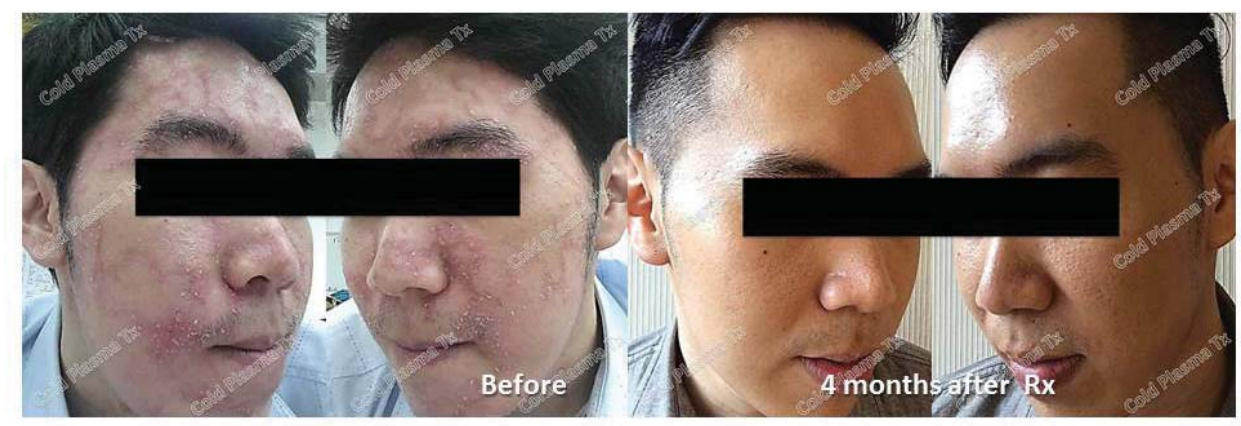

FIG. 7: Clearance of tinea faciei with 4-month follow-up (treatment once every 1-2 weeks)

surface complex, with visible changes in texture, pore size reduction, whitening, color, toning, fine lines, and mild to moderate skin tightening (Fig. 8).

\section{Herbal Cosmetic Synergistic Effect Enhancement}

Boonyawan et al. ${ }^{7}$ from Burapha University showed that direct DBD plasma from bioplasma synergistically enhanced the beneficial effects of herbal cosmetic products (Emblica Plus) in a study of 100 volunteers treated once weekly for 12 weeks (Fig. 9).

\section{Keratosis Pilaris}

Plasma peeling can also help relieve the problem of blocked hair follicles (keratosis pilaris). Successful clearance is observed after 2 weeks with two treatments (Fig. 10).

\section{Chronic Wound Management}

Chuangsuwanich et al. ${ }^{8}$ at Siriraj hospital, Mahidol University, conducted a study on grade 3 or 4 chronic pressure ulcers in 50 cases that did not improve within 3 weeks

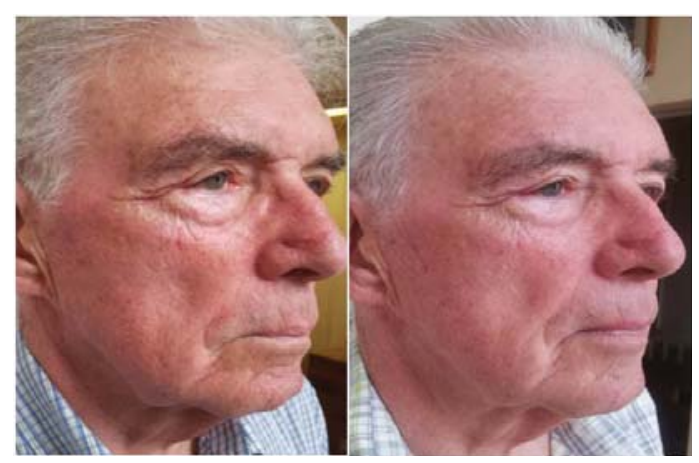

FIG. 8: Post bioplasma skin rejuvenation, 4-week follow-up after two treatments 


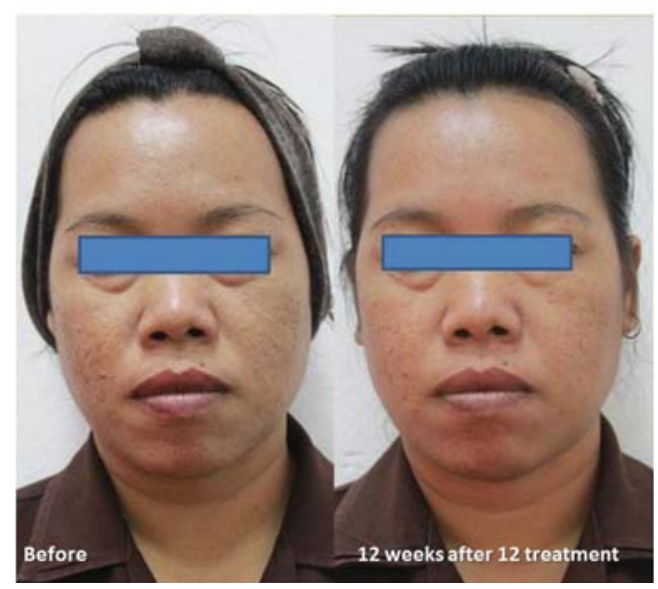

FIG. 9: Bioplasma and herbal cosmetic Emblica Plus

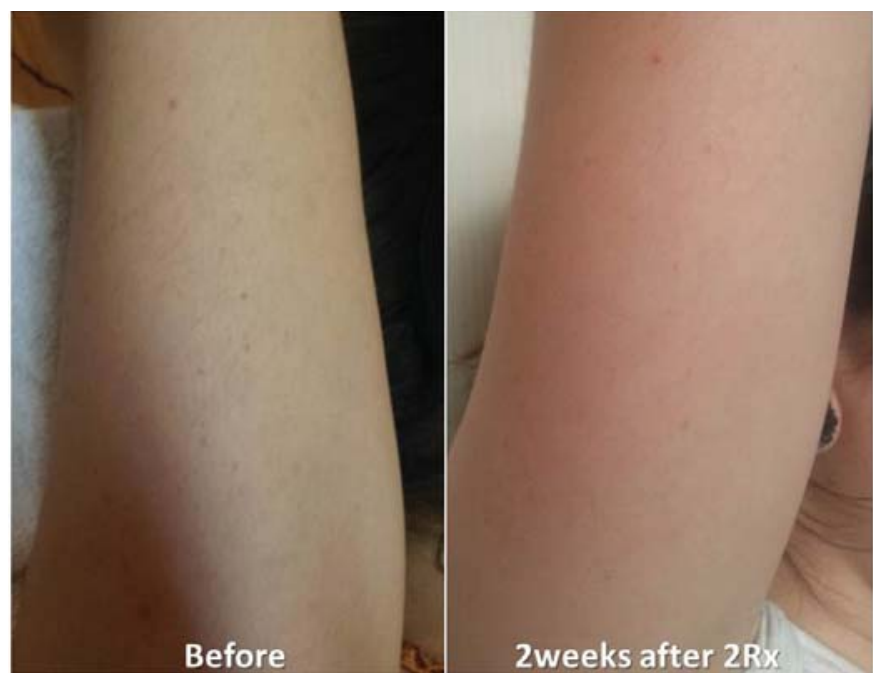

FIG. 10: Bioplasma peeling in keratosis pilaris

despite proper wound care. Randomized control trial with and without cold atmospheric plasma (CAP) in addition to conventional wound care. A noncontact non-thermal DBD direct with argon gas carrier was applied to the wound at 2-3 mm gap for 1 minute per square centimeter of lesion size, using a dynamic scan motion over the lesion. It was applied on the blind random selected cases after routine wound care once a week for 8 weeks. This was the first RCT study in vivo to demonstrate the wound healing effect of CAP. The CAP seems to support the clinical healing process of chronic pressure ulcers in humans. It reduced wound size and decreased exudate, improved the wound base, and reduced bacterial load within first few weeks. No relevant side effects have been observed with this method so far. The available outcomes from in vitro experiments and 

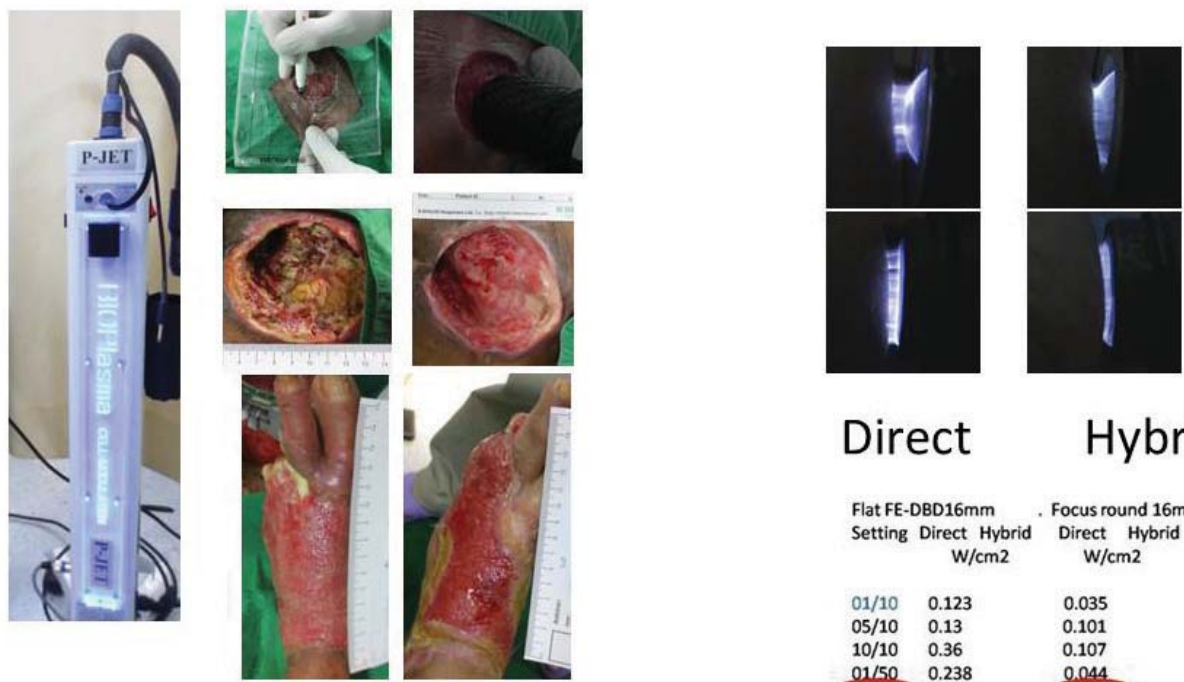

\section{Direct}

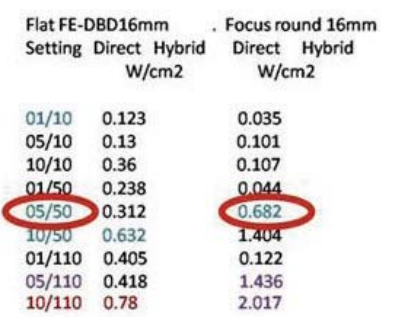

Application Direct DBD argon Bioplasma Jet

Slow scan noncontact motion on wound

$1-3 \mathrm{~mm}$ spacing : dose $1 \mathrm{minute} / \mathrm{sqcm}$ : $10 \mathrm{sqcm}=10 \mathrm{~min}$. Once

weekly as add on conventional $\mathrm{Rx}$

Figure6. Sterilizing effect without tissue damage $(0.6-2 \mathrm{~W} / \mathrm{cm} 2)$
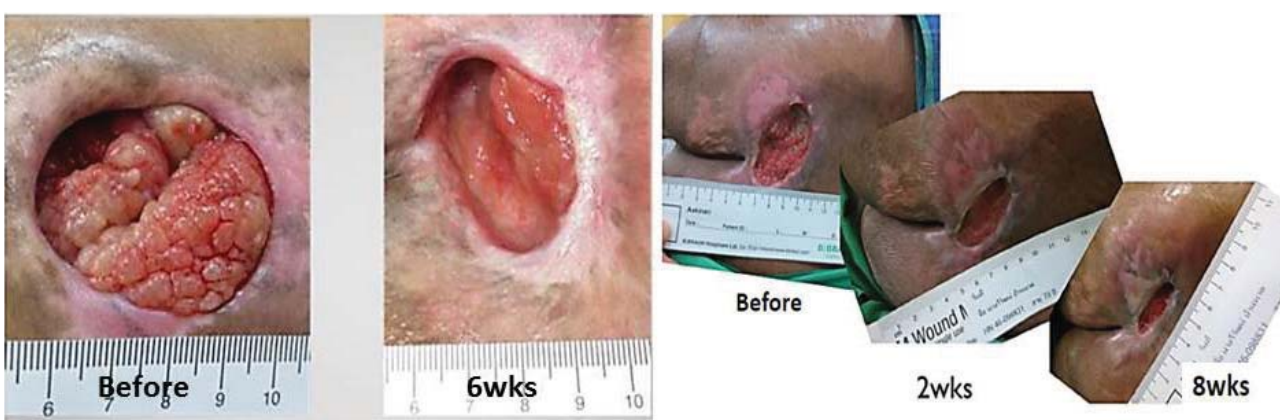

FIG. 11: Direct DBD, noncontact bioplasma argon jet in chronic pressure sore

these in vivo clinical results suggest that CAP is a promising new option for the treatment of chronic wounds (Fig. 11).

\section{DISCUSSION}

Plasma is defined as gas that contains ionized or charged particles. Plasma is different from normal gas because it is electrically conductive and responsive to electromagnetic energy. Plasma can be artificially created in normal atmospheric pressure and at the ambient room air conditions, which makes the biomedical applications of atmospheric plasma technology an interesting field for further exploration. Atmospheric plasma can be hot arcing plasma or low temperature cold plasma (non-thermal) depending on the

Volume 6, Issues 3-4, 2016 
degree of ionization, which affects the temperature of the gas associated with plasma stage. Electrosurgical devices (ESU) have been used for almost 100 years for surgical control of bleeding by electrothermal conductive and resistive heating. The electrical cutting and vaporizing of tissue also can be performed with high power density and high frequency continuous electrical waves. When emitted from a sharp or pointed electrode, it will induce electrical arcing at the tip of electrode with thermal mechanical disruption and cut deep into tissue to form a vaporizing gas. Another interesting function of ESU is spray coagulation, in which high voltage, low current electrical power is delivered in pulsed waves. This mode will create superficial wide spread tissue thermal coagulation, in which further spread of coagulation is limited because of the electrical property and thermal conductivity alteration after coagulation occurs. Argon plasma coagulation (APC) improves this spray coagulation mode, in which argon carrier gas and a floating electrode are used to help carry the hot plasma beam to tissue surface.

Recently, there have been significant developments in the application of non-thermal biological effects of electromagnetic energy, such as pulsed electric fields (electroporation), cold atmospheric pressure plasma (CAP) science, and engineering. New sources of CAP with well-controlled temperatures below $40^{\circ} \mathrm{C}$ have been designed, permitting safe plasma application for human use. In the last decade, a new innovative field, often referred to as plasma medicine, which combines plasma physics, life science, and clinical medicine has emerged. This field aims to exploit effects of partially ionized gas or cold plasma by controlling the interactions between plasma components (and other secondary species that can be formed from these components) with specific structural elements and functionalities of living cells. Recent studies showed that CAP can exert various beneficial effects, such as potent microbial eradication, modification of biological surface properties, stimulation of tissue regeneration, improved healing of chronic wounds, induction of tumor apoptosis, irreversible tissue denaturation, or high-dose tissue necrosis when applied according to the dosage specified by each generator. There is a wide safety dosage range with minimal toxicity to normal tissues. These features make plasma medicine another attractive future technology for exploration.

Our team in Thailand has started exploring the potential new applications using this low temperature and CAP or non-thermal plasma technology. A prototype electrical device with pulsing and super pulsing modes of high frequency and radiofrequency range has been developed as a plasma generator source. Special interest has been focused on developing DBD electrodes that can be designed to match the generator to produce various types of atmospheric plasmas, such as low temperature or non-thermal types. Direct, indirect, or hybrid applications can be used as contact or noncontact electrodes.

Clinical evaluation for tolerant dosage in human subjects showed that fixed exposure to argon gas at same spot for $15 \mathrm{sec}$ at $05 / 50 \mathrm{~Hz}$ will raise skin temperature to $39^{\circ} \mathrm{C}$. Associated plasma gas temperatures were measured and showed that with 6 min emission at a $5-\mathrm{mm}$ gap, the temperature will increase $11^{\circ} \mathrm{C}$, and with a $10-\mathrm{mm}$ gap, the maximum increase is $4^{\circ} \mathrm{C}$. 
The initial study reveals that moving the active electrode on skin with mild pressure will peel off the keratin layer and reduce the oily skin surface. Chutsirimongkol et al. ${ }^{1}$ demonstrated $75 \%$ reduction of $P$. acnes porphyrin bacterial count after 1 treatment at 1 week follow-up. Studies for acne treatment were carried out by the Mae Fah Luang University team (Fig. 6). These studies also revealed that skin texture and aesthetic skin property improved after acne treatment. Evaluation for this noninvasive atmospheric plasma for skin rejuvenation and aesthetic improvement were further explored. The study by Boonyawan et al. ${ }^{8}$ from Burapha University showed that this DBD direct contact air plasma also helped enhance the efficacy of a Thai herbal cosmetic product.

Extended studies were carried out at Siriraj Hospital, Mahidol University, for an RCT study of potential benefit of noncontact DBD argon bioplasma jet in chronic pressure wounds. The outcome showed safe and significant beneficial effect for chronic wound healing management (Fig. 11).

From the mentioned studies, it is quite clear that cold atmospheric plasma technology will have a significant role in medicine. Our team has further expanded our research and development to verify the potential new application in other biomedical fields.

\section{ACKNOWEDGMENIS}

We would like to extend our thanks to all plasma teams in Thailand, namely Plasma \& Beam Physics Research Facility (PBP) Faculty of Science/Chiangmai University (CMU), Mae Fah Luang (MFLU) University School of Antiaging, Faculty of Abhaibhubejhr Thai Traditional Medicine - Burapha University (BUU), Faculty of Medical Technology Rangsit University (RSU), Department of Surgery, and Faculty of Medicine Siriraj Hospital, Mahidol University, Thailand in contributing to this work. We also thank the National Research Council of Thailand (NRCT) and Thailand Center of Excellence for Life Science (TCELS) for grants supporting this project initiation in Thailand.

\section{REFERENCES}

1. Chutsirimongkol C, Boonyawan D, Polnikorn N, Techawatthanawisan W, Kundilokchai T. Non-thermal plasma for acne and aesthetic skin improvement. Plasma Med. 2014;4(1-4):79-88.

2. Thana Y, Ngamjarurojana A, Boonyawan D. Analysis of cold atmospheric-pressure bio-medicine plasmas by using UV absorption spectroscopy. Surf Coat Technol. 2016;306:106-12.

3. Yaopromsiri C, Yu LD, Sarapirom S, Thopan P, Boonyawan D. Effect of cold atmospheric pressure He-plasma jet on DNA change and mutation. Nucl Instrum Methods Phys Res B. 2015;365:399-403.

4. Kundilikchai T. The effectiveness of a non-thermal plasma device for reducing sebum production on oily face. Mae Fah Luang University, School of Anti-Aging and Regenerative Medicine: Annual Research Conference; Bangkok, Thailand. 2013.

5. Techawatthanawisan W. An efficacy assessment of a dielectric-barrier-discharge plasma device for adjunctive therapy of acne vulgaris: a pilot study; Mae Fah Luang University, School of Anti-Aging and Regenerative Medicine: Annual Research Conference; Bangkok, Thailand. 2012.

6. Bunsaisup C, Rummaneethorn P. A comparative study on the efficacy of non-thermal plasma device combined with topical treatment versus topical treatment alone for moderate facial acne vulgaris in

Volume 6, Issues 3-4, 2016 
Thais. 4th International Graduate Research Conference, Assumption University of Thailand; 2016 June 30-July 1; Bangkok, Thailand. 2016.

7. Boonyawan D, Kirdwichai W, Khemaleelakul S. Cold atmospheric plasma systems for medical and dental use. NRCT report. 2014.

8. Chuangsuwanich A, Assadamongkol T, Boonyawan D. The healing effect of low-temperature atmospheric-pressure plasma in pressure ulcer: A randomized controlled trial. Int J Low Extrem Wounds. 2016;15(4):313-9. 